\title{
Extracardiac findings on computed tomography attenuation correction: Is it worth paying extra attention?
}

\author{
Dominik C. Benz, MD, a and Tobias A. Fuchs, MD, FESC, FSCCT ${ }^{\mathrm{a}}$ \\ a Cardiac Imaging, Department of Nuclear Medicine, University Hospital Zurich, Zurich, Switzerland
}

Received Apr 20, 2017; accepted Apr 20, 2017

doi:10.1007/s12350-017-0907-y

\section{See related article, pp. 1574-1583}

Due to the profound impact of attenuation artifacts on diagnostic accuracy, especially specificity, ${ }^{1}$ several methods like computed tomography attenuation correction (CTAC), ${ }^{2}$ prone myocardial perfusion image (MPI) acquisition, ${ }^{3}$ or external radionuclide sources like ${ }^{153} \mathrm{Gd}^{4}$ have been developed. CTAC has evolved as the most common standard used, since it not only improves diagnostic accuracy and outcome prediction by reducing attenuation artifacts ${ }^{1,5,6}$ but also enables the evaluation of coronary calcium scoring, ${ }^{7}$ offering substantial added diagnostic and prognostic value. ${ }^{8,9}$ Furthermore, the application of a CTAC can discriminate defects caused by left bundle branch block from real perfusion defects in SPECT MPI ${ }^{10}$ and allows diagnosis of pulmonary hypertension by determining the diameter of the main pulmonary artery in CTAC. ${ }^{11}$

Moreover, incidental findings can be found in CTAC with a high prevalence. ${ }^{12,13}$ These early results on incidental findings in CTAC have recently been confirmed by a systematic review and meta-analysis demonstrating a prevalence of $44 \%$ incidental extracardiac findings, $16 \%$ major incidental extracardiac findings, and $0.7 \%$ previously unknown malignancies. ${ }^{14}$ Although most of these findings might be negligible, some are of clinical relevance. In fact, it is recommended by the EANM procedural guidelines for radionuclide MPI with SPECT and SPECT/CT that non-

\footnotetext{
Reprint requests: Tobias A. Fuchs, MD, FESC, FSCCT, Cardiac Imaging, Department of Nuclear Medicine, University Hospital Zurich, Raemistrasse 100, CH-8091, Zurich, Switzerland; tobias.fuchs@usz.ch

J Nucl Cardiol 2018;25:1584-7.

$1071-3581 / \$ 34.00$

Copyright (C) 2017 American Society of Nuclear Cardiology.
}

contrast-enhanced CT scans for AC and also scouts should be additionally screened by a physician fully trained in CT readings. ${ }^{15}$ Accordingly, the review of all visible non-cardiovascular structures is recommended by the Society of Cardiovascular Computed Tomography in patients undergoing non-contrast calcium scoring and coronary CT angiography. ${ }^{16}$ Despite these recommendations, the field still remains controversial. Considering the high prevalence of these findings, concerns are reported about increased cost and radiation exposure due to follow-up scans, morbidity of biopsy or resection, and anxiety of both the patient and the physician about a non-significant pathology. In addition, only a minority of incidental findings that seem clinically significant in CTAC might actually be detrimental to patient outcome. ${ }^{17}$ Hence, considering doubtful clinical relevance, the debate is ongoing whether it is worth paying extra attention to extracardiac findings.

In the current issue of the Journal of Nuclear Cardiology, Zadro et al. investigated the prevalence of extracardiac findings on CTAC for MPI by SPECT and evaluated the prognostic outcome. The authors retrospectively screened 1506 patients who underwent MPI SPECT and low-dose CTAC. Extracardiac findings of CTAC were captured and classified as major or minor abnormalities. The prevalence of minor and major extracardiac findings was high, which were found in $830(55.1 \%)$ and 212 (14.1\%) patients, respectively, whereas 464 (30.8\%) patients had no abnormality. Aneurysm of the aorta (33.8\%o), liver cirrhosis (27.2\%), non cystic hypodense lesions of the liver (18.5\%o), and suspicious nodules in the lung (16.6\%), accounted for the most frequent major abnormalities. Of note, in $53 \%$ of these patients, major extracardiac findings were previously unknown and $42 \%$ of these patients had an abnormal MPI SPECT.

Patients with major extracardiac findings were followed up over a period of $3.2 \pm 1.2$ years with an acceptable loss to follow-up rate $(5.2 \%)$. Among the patients with major extracardiac findings, 12 (6.0\%) died 
from cardiovascular vs $36(17.9 \%)$ from neoplastic causes, reflecting annual mortality rates of $1.9 \%$ vs $5.6 \%$, respectively $(p<0.001)$. This holds true for the subgroup of 103 patients with previously unknown major extracardiac findings. Neoplastic mortality was mainly due to bronchopulmonary cancer and hypodense intra-hepatic nodules reflecting hepatocellular carcinoma, metastasis, cholangiocarcinoma, and cancer of unknown primary syndrome. There was no significant impact on survival arising from abnormal myocardial perfusions SPECT, myocardial ischemia, or myocardial infarction scar in these patients.

Based on these results, the authors conclude that the frequency of extracardiac findings diagnosed by CTAC for SPECT MPI is high. There is no doubt that extracardiac findings are common in cardiac CT if the examiner looks beyond the heart, reinforcing what has been reported previously for non-contrast-enhanced low-resolution studies such as $\mathrm{CTAC}^{13,18}$ or coronary artery calcium scoring, ${ }^{19}$ and contrast-enhanced studies such as cardiac CT angiography, ${ }^{20}$ or cardiovascular CT prior to TAVI, ${ }^{21}$ or pulmonary vein isolation. ${ }^{22}$

The key question is whether the recognition of these non-cardiac findings translates into patient benefit or might even harm the patient. Yet potential benefits such as detection of cancer might outweigh the harms. The prevalence of previously unknown extracardiac malignancies of $1 \%$ reported by Zadro et al. is in line with $0.7 \%$ reported by Flor et al. ${ }^{14}$ However, on top, the study by Zadro et al. adds an important piece to the puzzle as it not only provides the prevalence of extracardiac findings, but also the outcome of these patients. The annual mortality rate of $10 \%$ reflects the significance of those findings. The authors should be congratulated for this comprehensive study investigating the follow-up of patients with major extracardiac findings over a time period of $3.2 \pm 1.2$ years. Unfortunately, the follow-up of patients without extracardiac findings was not assessed, which would have provided an important group for comparison.

So far, few studies have investigated the outcome of patients with extracardiac findings in patients undergoing cardiac CT. Lindsay et al. who investigated the consequences of incidental non-cardiac findings detected on cardiovascular CT in 279 patients planned for TAVI found that survival was significantly associated with the presence of an extracardiac finding. However, in multivariate analysis, outcomes after TAVI were not influenced by any category of incidental finding. ${ }^{21}$ Additionally, a retrospective observational study including 414 patients undergoing CT prior to transcatheter aortic valve replacement detected a potentially malignant incidental finding in $19 \%$ of patients-with no impact on 2-year survival. ${ }^{23}$ Although these patient cohorts differ significantly from those undergoing MPI by SPECT taking into account the high mortality rate of patients with severe aortic stenosis. Furthermore, acquisition protocols offer better image quality compared to attenuation correction scans and thereby might reduce the number of false positive findings.

Regarding incidental extracardiac findings on CTAC for MPI by SPECT, a prospective multicentre study investigating 1819 patients recently reported that out of $51(2.8 \%)$ patients with clinically significant extracardiac findings, only 4 patients $(0.2 \%)$ had a clinically significant pathology. ${ }^{18}$ Two years later, adding a further 1672 patients, the same research group concluded that routine reporting of CTAC images is not beneficial due to a low positive predictive value over a 2-year period of $12 \% .^{24}$

Conversely, the detrimental impact of incidental findings on clinical outcome has been documented by a recent study that retrospectively identified 135 patients $(12 \%)$ with incidental findings in CTAC. ${ }^{25}$ Although these findings did not significantly increase all-cause mortality, they were associated with a significantly higher cancer-specific mortality-indicating that patients with incidental findings may more often end up with lethal cancer. Nonetheless, it is not self-evident that the detection of an incidental finding triggering instant work-up and appropriate treatment will prolong survival. Moreover, in screening examinations, a so-called lead-time bias might appear to increase survival time by early diagnosis, but in fact, no additional life span has been gained.

Heterogeneity of the above-mentioned study results might partially be contributed to by the huge variability of scanning protocols for CTAC. Zadro et al. scanned with a slice thickness of $2.5 \mathrm{~mm}$ during inspiration breath-hold, Qureshi et al. used a 8-slice non-gated CT with a slice thickness of $3.75 \mathrm{~mm}$ during free breathing, ${ }^{25}$ and the initially reported high variance of the positive predictive value regarding extracardiac findings might reflect different scan parameters such as singleslice CT vs. 16-slice CT and an acquired slice thickness of $10 \mathrm{~mm}$ during free breathing vs $1.5 \mathrm{~mm}$ during breath-hold in the four centres of the study by Coward et al. $^{18}$ Due to technical refinements, acquisition parameters have continuously improved. Meanwhile, even 64-slice CT is used during inspirational breath-hold to acquire CTAC. ${ }^{2}$ This not only enables the simultaneous use of these scans for calcium scoring, ${ }^{7}$ but might also improve diagnostic accuracy of incidental extracardiac findings. However, despite further technical refinements, due to the lower resolution and limited scanned area of the thorax, even though a pathology might be detected on the images, the absence of pathology on CTAC does not exclude disease, simply 
because a pathology outside the scan range will invariably remain undetected.

Finally, the evidence on the prevalence of incidental extracardiac findings in CTAC images is staggering and the association with poor outcome-even independent of the MPI findings-was demonstrated in the present study. Although the clinical relevance of extracardiac findings remains in debate, the present study underpins the guidelines' recommendations to review all visible non-cardiovascular structures and strengthens the physician's certainty that the chance of detecting a clinically relevant extracardiac finding that might have a significant impact on the patient's survival outweighs the risk of physical and financial harm due to additional testing. Additionally, looking not only beyond the heart but also beyond the whole image, e.g., assessing clinical history or risk factors and interdisciplinary discussion with the referring physician, might further improve accurate assessment of extracardiac findings.

\section{Disclosure}

There are no conflicts of interest to disclose related to this article for all authors.

\section{References}

1. Huang JY, Huang CK, Yen RF, Wu HY, Tu YK, Cheng MF, et al. Diagnostic performance of attenuation-corrected myocardial perfusion imaging for coronary artery disease: a systematic review and meta-analysis. J Nucl Med. 2016;57:1893-8.

2. Herzog BA, Buechel RR, Husmann L, Pazhenkottil AP, Burger IA, Wolfrum M, et al. Validation of CT attenuation correction for high-speed myocardial perfusion imaging using a novel cadmiumzinc-telluride detector technique. J Nucl Med. 2010;51:1539-44.

3. Nishina H, Slomka PJ, Abidov A, Yoda S, Akincioglu C, Kang X, et al. Combined supine and prone quantitative myocardial perfusion SPECT: method development and clinical validation in patients with no known coronary artery disease. J Nucl Med. 2006; 47:51-8

4. Tan P, Bailey DL, Meikle SR, Eberl S, Fulton RR, Hutton BF. A scanning line source for simultaneous emission and transmission measurements in SPECT. J Nucl Med. 1993;34:1752-60.

5. Malkerneker D, Brenner R, Martin WH, Sampson UK, Feurer ID, Kronenberg MW, et al. CT-based attenuation correction versus prone imaging to decrease equivocal interpretations of rest/stress Tc-99 m tetrofosmin SPECT MPI. J Nucl Cardiol. 2007;14:31423.

6. Pazhenkottil AP, Ghadri JR, Nkoulou RN, Wolfrum M, Buechel RR, Küest SM, et al. Improved outcome prediction by SPECT myocardial perfusion imaging after CT attenuation correction. J Nucl Med. 2011;52:196-200.

7. Schepis T, Gaemperli O, Koepfli P, Rüegg C, Burger C, Leschka $\mathrm{S}$, et al. Use of coronary calcium score scans from stand-alone multislice computed tomography for attenuation correction of myocardial perfusion SPECT. Eur J Nucl Med Mol Imaging. 2007;34:11-9.
8. Schepis T, Gaemperli O, Koepfli P, Namdar M, Valenta I, Scheffel $\mathrm{H}$, et al. Added value of coronary artery calcium score as an adjunct to gated SPECT for the evaluation of coronary artery disease in an intermediate-risk population. $\mathrm{J}$ Nucl Med. 2007;48:1424-30.

9. Engbers EM, Timmer JR, Ottervanger JP, Mouden M, Knollema S, Jager PL. Prognostic value of coronary artery calcium scoring in addition to single-photon emission computed tomographic myocardial perfusion imaging in symptomatic patients. Circ Cardiovasc Imaging. 2016;9(5):e003966.

10. Hoefflinghaus T, Husmann L, Valenta I, Moonen C, Gaemperli O, Schepis $\mathrm{T}$, et al. Role of attenuation correction to discriminate defects caused by left bundle branch block versus coronary stenosis in single photon emission computed tomography myocardial perfusion imaging. Clin Nucl Med. 2008;33:748-51.

11. Burger IA, Husmann L, Herzog BA, Buechel RR, Pazhenkottil AP, Ghadri JR, et al. Main pulmonary artery diameter from attenuation correction CT scans in cardiac SPECT accurately predicts pulmonary hypertension. J Nucl Cardiol. 2011;18:634-41.

12. Osman MM, Cohade C, Fishman EK, Wahl RL. Clinically significant incidental findings on the unenhanced CT portion of PET/CT studies: frequency in 250 patients. J Nucl Med. 2005;46:1352-5.

13. Husmann L, Tatsugami F, Aepli U, Herzog BA, Valenta I, VeitHaibach $\mathrm{P}$, et al. Prevalence of noncardiac findings on low dose 64-slice computed tomography used for attenuation correction in myocardial perfusion imaging with SPECT. Int $\mathrm{J}$ Cardiovasc Imaging. 2009;25:859-65.

14. Flor N, Di Leo G, Squarza SA, Tresoldi S, Rulli E, Cornalba G, et al. Malignant incidental extracardiac findings on cardiac CT: systematic review and meta-analysis. AJR Am J Roentgenol. 2013;201:555-64.

15. Verberne HJ, Acampa W, Anagnostopoulos C, Ballinger J, Bengel $\mathrm{F}$, De Bondt $\mathrm{P}$, et al. EANM procedural guidelines for radionuclide myocardial perfusion imaging with SPECT and SPECT/CT: 2015 revision. Eur J Nucl Med Mol Imaging. 2015;42(2):1929-40.

16. Leipsic J, Abbara S, Achenbach S, Cury R, Earls JP, Mancini GJ, et al. SCCT guidelines for the interpretation and reporting of coronary CT angiography: a report of the Society of Cardiovascular Computed Tomography Guidelines Committee. J Cardiovasc Comput Tomogr. 2014;8:342-58.

17. Budoff MJ, Fischer H, Gopal A. Incidental findings with cardiac CT evaluation: should we read beyond the heart? Catheter Cardiovasc Interv. 2006;68:965-73.

18. Coward J, Lawson R, Kane T, Elias M, Howes A, Birchall J, et al. Multi-centre analysis of incidental findings on low-resolution CT attenuation correction images. Br J Radiol. 2014;87:20130701.

19. Gil BN, Ran K, Tamar G, Shmuell F, Eli A. Prevalence of significant noncardiac findings on coronary multidetector computed tomography angiography in asymptomatic patients. J Comput Assist Tomogr. 2007;31:1-4.

20. Johnson KM, Dennis JM, Dowe DA. Extracardiac findings on coronary CT angiograms: limited versus complete image review. AJR Am J Roentgenol. 2010;195:143-8.

21. Lindsay AC, Sriharan M, Lazoura O, Sau A, Roughton M, Jabbour $\mathrm{RJ}$, et al. Clinical and economic consequences of non-cardiac incidental findings detected on cardiovascular computed tomography performed prior to transcatheter aortic valve implantation (TAVI). Int J Cardiovasc Imaging. 2015;31:1435-46.

22. Sohns JM, Menke J, Staab W, Spiro J, Fasshauer M, Kowallick JT, et al. Current role of cardiac and extra-cardiac pathologies in clinically indicated cardiac computed tomography with emphasis on status before pulmonary vein isolation. Rofo. 2014;186:860-7.

23. Stachon P, Kaier K, Milde S, Pache G, Sorg S, Siepe M, et al. Two-year survival of patients screened for transcatheter aortic 
valve replacement with potentially malignant incidental findings in initial body computed tomography. Eur Heart $\mathrm{J}$ Cardiovasc Imaging. 2015;16:731-7.

24. Coward J, Lawson R, Kane T, Elias M, Howes A, Birchall J, et al. Multicentre analysis of incidental findings on low-resolution CT attenuation correction images: an extended study. Br J Radiol. 2015;88:20150555.

25. Qureshi WT, Alirhayim Z, Khalid F, Al-Mallah MH. Prognostic value of extracardiac incidental findings on attenuation correction cardiac computed tomography. J Nucl Cardiol. 2016;23:1266-74. 